\title{
Simple diffusion delivery via brain interstitial route for the treatment of cerebral ischemia
}

\author{
HAN HongBin ${ }^{1 *}$, XIA ZuoLi $^{2 *}, \mathrm{CHEN} \mathrm{He}^{1}, \mathrm{HOU} \mathrm{Chao}^{1} \& \mathrm{LI} \mathrm{WeiBo}^{3}$ \\ ${ }^{1}$ Department of Radiology, Peking University Third Hospital, Beijing 100191, China; \\ ${ }^{2}$ Department of Neurology, Affiliated Hospital, Taishan Medical College, Tai'an 271000, China; \\ ${ }^{3}$ Department of Medical Radiation Physics and Diagnostics, Helmholtz Zentrum München, German Research Center for \\ Environmental Health (GmbH), Neuherberg 85764, Germany
}

Received December 13, 2010; accepted December 20, 2010; published online February 19, 2011

\begin{abstract}
Delivering pharmacologic agents directly into the brain has been proposed as a means of bypassing the blood brain barrier. However, despite 16 years of research on a number of central nervous system disorders, an effective treatment using this strategy has only been observed in the brain tumor glioblastoma multiforme. Within this study we propose a novel system for delivering drugs into the brain named the simple diffusion (SDD) system. To validate this technique, rats were subjected to a single intracranial (at the caudate nucleus), or intraperitoneal injection, of the compound citicoline, followed two hours later by a permanent middle cerebral artery occlusion (pMCAO). Results showed that $12 \mathrm{~h}$ after pMCAO, with $0.0025 \mathrm{~g} \mathrm{~kg}^{-1}$ citicoline, an infarct volume 1/6 the size of the intraperitoneal group was achieved with a dose $1 / 800$ of that required for the intraperitoneal group. These results suggest that given the appropriate injection point, through SDD a pharmacologically effective concentration of citicoline can be administered.
\end{abstract}

permanent middle cerebral artery occlusion, animal model, brain ischemic injury, citicoline therapy, MRI, neuroprotection

Citation: Han H B, Xia Z L, Chen H, et al. Simple diffusion delivery via brain interstitial route for the treatment of cerebral ischemia. Sci China Life Sci, 2011, 54: 235-239, doi: 10.1007/s11427-011-4141-6

A major obstacle in the development of drugs for use in the central nervous system is the ability to generate compounds capable of penetrating the blood brain barrier (BBB) [1]. One strategy, developed by Oldfield et al. [2], to overcome this problem is to bypass the $\mathrm{BBB}$ and infuse drugs directly into the CNS parenchyma through a process known as convection-enhanced delivery. With this technique a constant pressure gradient is maintained during interstitial infusion via one or several catheters and there is significantly enhanced distribution of a range of molecule sizes and an increase in the locoregional concentration of the infused compounds. Despite 16 years of basic research and multiple clinical trials using this technique being carried out on

*Corresponding author (email: hanhongbin@bjmu.edu.cn; xiazuoli@163.com) various CNS diseases [3], effective treatment has only been obtained in the brain tumor glioblastoma multiforme [4].

In this report, we outline the findings of a novel simple diffusion delivery (SDD) system used for the treatment of cerebral ischemia. This system is based on previous studies examining brain interstitial tracer imaging using MRI [5] wherein, we demonstrated that within two hours, using the caudate nucleus as the original injection point, small molecules like Gd-DTPA are able to diffuse via the brain extracellular space to most of the architecture supplied by the middle cerebral artery. The compound Gd-DTPA is a common MRI contrast agent, which is also an effective tracer for monitoring the dynamic distribution of small molecules in the interstitial space [6]. Based on the above findings, we sought to test the hypothesis that intracerebral 
administration of the compound citicoline prior to ischemic injury would provide a more efficient neuroprotective effect than other routes of administration. In the current study, citicoline was chosen to test our hypothesis because it has a similar polarity and molecular weight to Gd-DTPA $[7,8]$.

\section{Materials and methods}

\subsection{Animals}

The study was conducted in accordance with national guidelines for the care and use of animals. All animal protocols were approved by the Peking University Health Science Center Ethics Committee (Approval No. LA2009-008). The animals used in the study were age-matched, mature male Sprague Dawley rats weighing approximately 250$300 \mathrm{~g}$.

\subsection{Experimental groups}

Citicoline (CDP-choline, cytidine 5'-diphosphocholine) was administered by intracranial microinjection into the caudate nucleus using the following stereotactic coordinates: bregma +1 $\mathrm{mm}$, lateral $3 \mathrm{~mm}$, vertical $4.5 \mathrm{~mm}$ (Lab Standard Stereotaxic-Single, Stoelting Co., Illinois, USA). The study of the real-time detection of the infarction process by MRI and determination of infarct volume by TTC staining was composed of seven groups. For each group the animals ( $n=$ 8/group) received an intracranial (IC) microinjection of (i) $5 \mu \mathrm{L}$ saline (control group), (ii) $0.00125 \mathrm{~g} \mathrm{~kg}^{-1}$ citicoline (IC group 1), (iii) $0.0025 \mathrm{~g} \mathrm{~kg}^{-1}$ citicoline (IC group 2), (iv) $0.00375 \mathrm{~g} \mathrm{~kg}^{-1}$ citicoline (IC group 3), (v) $0.005 \mathrm{~g} \mathrm{~kg}^{-1}$ citicoline (IC group 4), (vi) $0.0125 \mathrm{~g} \mathrm{~kg}^{-1}$ citicoline (IC group 5). Animals in the final group received an intraperitoneal (IP) injection of $2 \mathrm{~g} \mathrm{~kg}^{-1}$ citicoline (IP group). Two hours after receiving an injection of citicoline (or saline), the animals were then subjected to a permanent middle cerebral artery occlusion (pMCAO). The choice to administer citicoline two hours prior to pMCAO was based on our previous study on the diffusion pattern of Gd-DTPA in the brain extracellular space [5].

\subsection{Permanent middle cerebral artery occlusion (pMCAO) in rats}

The rats were anesthetized with a compound anesthetic agent (sodium pentobarbital, ethanol, chloral hydrate, magnesium sulfate, propylene glycol) delivered intraperitoneally. The animals' core temperatures were monitored with a rectal thermometer and maintained at approximately $(38 \pm 0.5)^{\circ} \mathrm{C}$ using a heat pad. Permanent focal cerebral ischemia was induced using a technique modified from work presented by Longa et al. [9] without the process of vascular recanaliza- tion for perfusion. After the surgery, all animals were returned to their cages and allowed free access to water and food.

\subsection{MRI for pMCAO rats}

Neuroimaging was performed using a 3.0T clinical MR system (Magnetom Trio, Siemens Medical Solutions, Erlangen, Germany). A wrist coil was used for axial $\mathrm{T}_{1} \mathrm{~W}$, $\mathrm{T}_{2} \mathrm{~W}$ and DW images. Parameters for the imaging sequence were as follows: (i) 3D MP-RAGE $\mathrm{T}_{1} \mathrm{WI}$ (echo time $(\mathrm{TE})=$ $3.7 \mathrm{~ms}$; repetition time $(\mathrm{TR})=1500 \mathrm{~ms}$; flip angle $\alpha=9^{\circ}$; inversion time $(\mathrm{TI})=900 \mathrm{~ms}$; field of view $(\mathrm{FOV})=267 \mathrm{~mm}$; voxel=0.5 mm $\times 0.5 \mathrm{~mm} \times 0.5 \mathrm{~mm}$; resolution=512 $\times 512$ ); (ii) $\mathrm{TSE}_{2} \mathrm{~T}$ W $\left(\mathrm{TE}=92 \mathrm{~ms} ; \mathrm{TR}=3620 \mathrm{~ms}\right.$; flip angle $\alpha=120^{\circ}$; slice thickness, $2 \mathrm{~mm}$; FOV=80 mm; resolution=256 $\times 256$ ); (iii) FSIP-DWI (TE $=6.22 \mathrm{~ms}$; TR $=15.3 \mathrm{~ms}$; flip angle $\alpha=30^{\circ}$; slice thickness, $1 \mathrm{~mm}$; FOV $=80 \mathrm{~mm}$; voxel $=0.4 \mathrm{~mm} \times 0.4$ $\mathrm{mm} \times 1.0 \mathrm{~mm}$; diffusion moment $=0$ and $50 \mathrm{mT} \mathrm{m} \mathrm{ms}^{-1}$; resolution=192 $\times 192$ ). MRI scanning was performed $12 \mathrm{~h}$ after the pMCAO surgery and prior to sacrifice.

\subsection{Neurological deficit evaluation}

A thorough neurologic examination was performed on all animals after surgery. The neurologic findings were scored along a five-point scale based on the Zea-Longa scale [7] whereby zero indicated no neurologic deficit, a score of one (characterized by the failure to extend left forepaw fully) indicated a mild focal neurologic deficit, a score of two (characterized by circling to the left) indicated a moderate focal neurologic deficit and a score of three (characterized by falling to the left) indicated a severe focal deficit. Rats with a score of four did not walk spontaneously and had a depressed level of consciousness.

\subsection{TTC staining and infarct volume determination}

Twelve hours after performing the pMCAO surgery the animals were sacrificed. The brains were removed and series of $2 \mathrm{~mm}$ of coronal brain slices were collected (Brain Matrix, EHSY, Shanghai, China) and stained for $30 \mathrm{~min}$ at $37^{\circ} \mathrm{C}$ in $0.2 \%$ TTC (2,3,5-triphenyl-tetrazolium chloride, Sigma, Missouri, USA) diluted in $0.1 \mathrm{~mol} \mathrm{~L}^{-1}$ phosphate buffer [10]. The infarct volume was measured by recording stained sections with a digital camera (Canon IXUS800, Canon, Tokyo, Japan) and the image of each section was analyzed by an image analyzer (Image Pro Plus 6.0, Media Cybernetics, Inc., Georgia, USA). The infarct area, which was not stained, was determined by counting pixels within the outlined regions of interest. Infarct volume was expressed as the ratio of pixels counted from the corrected infarct area to the pixels collected from the whole contralateral hemisphere. We then compared the infarct regions with those derived from MRI. 


\subsection{Statistical analysis}

Values are presented as mean \pm SD. Statistical analysis was performed using SPSS 13.0. One-way analysis of variance followed by individual comparisons of means (LSD test, or Dunnet's method when the data were not normally distributed) was used for the comparison of grouped data. Data showing $P<0.05$ were considered statistically significant.

\section{Results}

\subsection{Intracranial administration of citicoline reduces infarct volume and neurological deficit of brain ischemic injury}

The results showed that IC administration of citicoline prior to pMCAO had a neuroprotective effect. These effects were confirmed at both the pathological (via TTC staining) and functional levels (via a Zea-Longa five-point scale). The best effect was observed in IC group 2 relative to all other groups $(P<0.01$, Table 1 and Figures 1 and 2$)$. There was no statistical difference between IC groups 1, 3, 4 and 5, the IP group and the control group $(P>0.05$, Table 1 and Figures 1 and 2). On the other hand, there was a statistical difference between IC group 2 and the IP group $(P<0.01$, Table 1 and Figures 1 and 2). As observed in a previous study, behavioral deficits were correlated to the severity of the damage in the cortex [9]. Therefore, the improved neural function might be attributed to a rescue of the penumbra in external cortex by citicoline administration, which was confirmed by the MRI findings (Figure 2).

\subsection{MRI results at $12 \mathrm{~h}$ after pMCAO}

We also used MRI to monitor the pathological progression of all groups during ischemia. Generally, $\mathrm{T}_{1} \mathrm{WI}$ is good for anatomical imagine whereas $\mathrm{T}_{2} \mathrm{WI}$ is known to be highly sensitive to water content changes. The typical MRI changes associated with infarction, including decreased signal intensity on $\mathrm{T}_{1} \mathrm{WI}$ and increased signal intensity on $\mathrm{T}_{2} \mathrm{WI}$ [11], are presented to define the lesions for both localization and injury severity (Figure 2).

The TTC staining and MRI showed different localizations of ischemic lesions in six of the groups. The typical changes of decreased signal intensity on $\mathrm{T}_{1} \mathrm{WI}$ and increased signal intensity on $\mathrm{T}_{2} \mathrm{WI}$, were well displayed in the infarct areas. Each group consisted of $n=8$ animals.

\section{Discussion}

In the present work, we have developed a simple diffusion delivery (SDD) technique, which has the potential to distribute the pharmacological agents more efficiently and less invasively within the brain parenchyma. Using this technique, a significant neuroprotective effect against cerebral ischemia can be achieved with a single injection of only a very small dose of the agent. The efficiency of SDD is based on the original injection point and the neuroprotective agents used.

The design of the SDD system was based on brain interstitial MRI tracer imaging using the gadolinium derivative Gd-DTPA whereby MRI can be used to trace the diffusion and distribution of Gd-DTPA within the brain interstitial space. Our previous study found that using the caudate nucleus as the original injection point a single administration Gd-DTPA was able to diffuse to the MCA territory and where it remained almost unchanged for $2-4 \mathrm{~h}$ after the injection [5]. In the current work, we assumed that a neuroprotective agent similar to Gd-DTPA would diffuse in the same way as Gd-DTPA. Among the many candidates, citicoline was chosen because it is similar to Gd-DTPA in respect to both molecular weight (510.31 Da vs. 938.02 Da) and polarity (both are hydrophilic). Additionally, because citicoline is an intermediate in the generation of phosphatidylcholine from choline in the normal brain, it is likely to be safer and more appropriate than other exogenous compounds for this local intracranial drug delivery study [12].

Citicoline is considered to be one of the most promising candidates for the treatment of acute ischemic stroke [13-16]. Its functions include accelerating resynthesis of phospholipids and suppressing the release of free fatty acids, stabilizing cell membranes and reducing free radical generation [17]. However, the extremely low brain uptake of citicoline is one of the major obstacles affecting its efficacy as demonstrated in meta-analysis of clinical trials assessing the concentration-dependent neuroprotective effects of citicoline [18]. More importantly, the limited volume fraction of brain microvessels (3\% of the total brain volume) [19], and the post-ischemic hypoperfusion state before successful vascular recanalization therapy also make the infusion of citicoline to the infarct area even more difficult. In the present work, using SDD after a single administration of small dose of citicoline resulted in significant protective effects. Our results indicate that citicoline successfully circumvented the BBB and suggests that the issue of poor delivery

Table 1 Neurological findings (based on a five-point Zea-Longa score) and infarct volume ratio observed $12 \mathrm{~h}$ after pMCAO surgery ${ }^{\mathrm{a}}$

\begin{tabular}{|c|c|c|c|c|c|c|c|}
\hline Groups & $\begin{array}{l}\text { Control group } \\
\text { (saline) }\end{array}$ & $\begin{array}{c}\text { IC group } 1 \\
\left(0.00125 \mathrm{~g} \mathrm{~kg}^{-1} \text { citicoline }\right)\end{array}$ & IC group 2 & IC group 3 & IC group 4 & IC group 5 & IP group \\
\hline Neurological score & $2.9 \pm 0.8$ & $2.9 \pm 0.8$ & $1.6 \pm 0.9$ & $1.9 \pm 0.4$ & $2.0 \pm 1.2$ & $1.7 \pm 0.6$ & $2.0 \pm 0.8$ \\
\hline Infarct volume ratio $(\%)$ & $27.7 \pm 10.5$ & $27.5 \pm 11.9$ & $4.1 \pm 2.0$ & $24.0 \pm 12.9$ & $23.5 \pm 11.7$ & $30.3 \pm 18.8$ & $24.0 \pm 10.4$ \\
\hline
\end{tabular}

a) Animals from IC group 6 did not survive $12 \mathrm{~h}$ and were not included. For each group $n=8$ rats. 


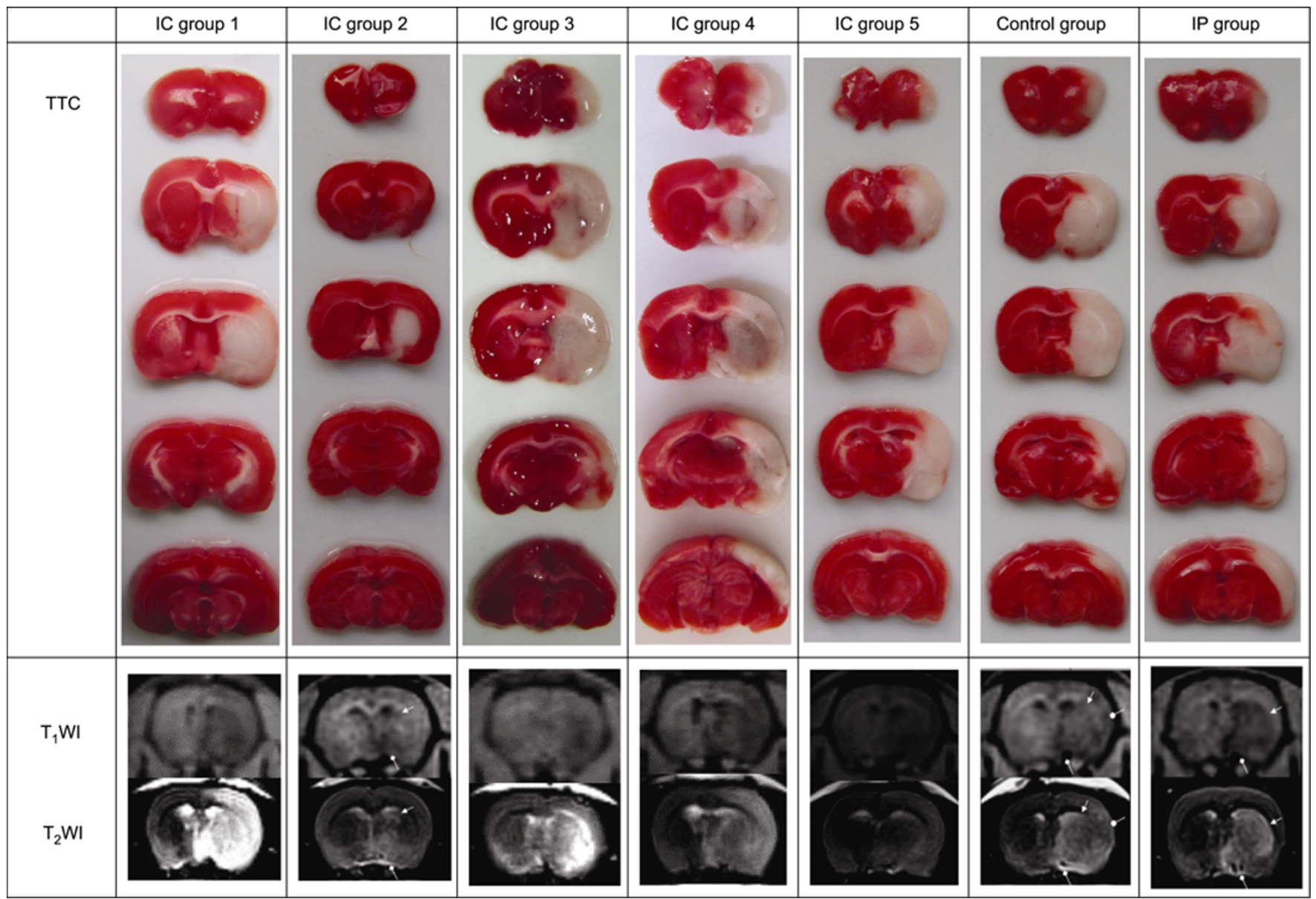

Figure 1 TTC staining and MRI results. Each group, except group 7, received an IC microinjection containing $5 \mu \mathrm{L}$ saline (group 1, control group), $0.00125 \mathrm{~g} \mathrm{~kg}^{-1}$ citicoline (group 2, IC group 1), $0.0025 \mathrm{~g} \mathrm{~kg}^{-1}$ citicoline (group 3, IC group 2), $0.00375 \mathrm{~g} \mathrm{~kg}^{-1}$ citicoline (group 4, IC group 3), $0.005 \mathrm{~g} \mathrm{~kg}^{-1}$ citicoline (group 5, IC group 4), and $0.0125 \mathrm{~g} \mathrm{~kg}^{-1}$ citicoline (group 6, IC group 5). Animals from group 7 (IP group) received an IP injection of $2 \mathrm{~g} \mathrm{~kg}^{-1}$ citicoline. For each group, two hours following injection, the rats received a permanent middle cerebral artery occlusion (pMCAO).

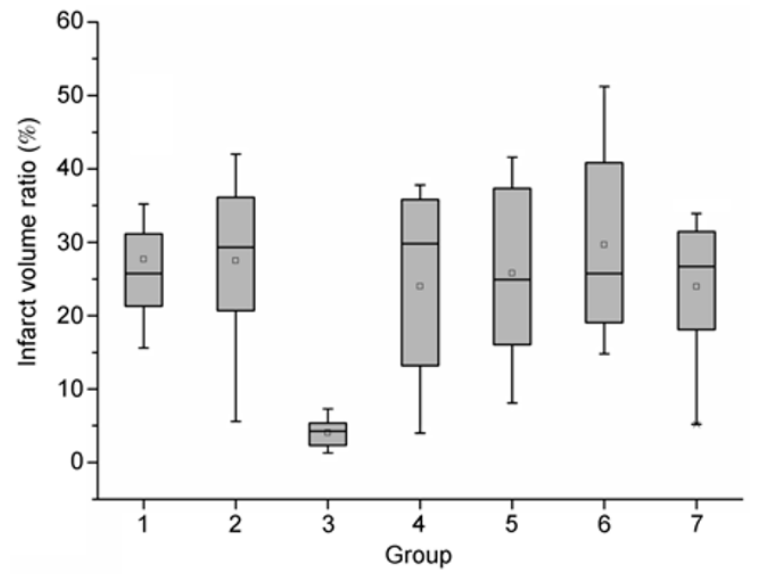

Figure 2 The infarct volume ratio in the seven groups. Each group, except group 7, received an IC microinjection containing $5 \mu \mathrm{L}$ saline (group 1, control group), $0.00125 \mathrm{~g} \mathrm{~kg}^{-1}$ citicoline (group 2, IC group 1), $0.0025 \mathrm{~g} \mathrm{~kg}^{-1}$ citicoline (group 3, IC group 2), $0.00375 \mathrm{~g} \mathrm{~kg}^{-1}$ citicoline (group 4, IC group 3), $0.005 \mathrm{~g} \mathrm{~kg}^{-1}$ citicoline (group 5, IC group 4), and $0.0125 \mathrm{~g} \mathrm{~kg}^{-1}$ citicoline (group 6, IC group 5). Animals from group 7 (IP group) received an IP injection of $2 \mathrm{~g} \mathrm{~kg}^{-1}$ citicoline. For each group, two hours following injection, the rats received a permanent middle cerebral artery occlusion (pMCAO). Each group consisted of $n=8$ animals. due to hypoperfusion may have also been overcome.

In summary, we have developed a novel delivery technique for the treatment of CNS diseases. Compared with traditional techniques such as convection-enhanced delivery, SDD is more efficient and less invasive. Although the MRI tracer imaging enables a precise predictive control over the SDD process in our study, a reliable quantitative measurement on the diffusion and clearance properties within brain interstitial space is the key to understanding the mechanism of SDD and enhancing its application for the treatment of CNS diseases.

This work was supported by the National Natural Science Foundation of China (Grant Nos. 30972811 and 81071148) and Natural Science Foundation of Beijing (Grant No. 7093137). We thank Professor Dai JianPing and Liu XingDi for their suggestions regarding experimental design.

1 Fisher M, Feuerstein G, Howells D W, et al. Update of the stroke therapy academic industry roundtable preclinical recommendations. Stroke, 2009, 40: 2244-2250

2 Bobo R H, Laske D W, Akbasak A, et al. Convection-enhanced delivery of macromolecules in the brain. Proc Natl Acad Sci USA, 1994, 
91: 2076-2080

3 Debinski W, Tatter S B. Convection-enhanced delivery for the treatment of brain tumors. Expert Rev Neurother, 2009, 9: 1519-1527

4 Gallia G L, Brem S, Brem H. Local treatment of malignant brain tumors using implantable chemotherapeutic polymers. J Natl Compr Cancer Netw, 2005, 3: 721-728

$5 \mathrm{He} \mathrm{Q}$, Han $\mathrm{H}, \mathrm{Xu} \mathrm{F}$, et al. Imaging and quantitative measurement of brain extracellular space using MRI Gd-DTPA tracer method. J Peking Univ (Health Sci), 2010, 42: 188-191

6 Krauze M T, Forsayeth J, Park J W, et al. Real-time imaging and quantification of brain delivery of liposomes. Pharm Res, 2006, 23 : 2493-2504

7 Fresta M, Puglisi G, Di Giacomo C, et al. Liposomes as in vivo carriers for citicoline: Effects on rat cerebral post-ischemic reperfusion. J Pharm Pharmacol, 1994, 46: 974-981

8 Caravan P, Ellison J J, McMurry T J, et al. Gadolinium(iii) chelates as MRI contrast agents: Structure, dynamics, and applications. Chem Rev, 1999, 99: 2293-2352

9 Longa E Z, Weinstein P R, Carlson S, et al. Reversible middle cerebral artery occlusion without craniectomy in rats. Stroke, 1989, 20: 84-91

10 Joshi C N, Jain S K, Murthy P S. An optimized triphenyltetrazolium chloride method for identification of cerebral infarcts. Brain Res Brain Res Protoc, 2004, 13: 11-17
11 Moseley M E, Cohen Y, Mintorovitch J, et al. Early detection of regional cerebral ischemia in cats: Comparison of diffusion- and T2-weighted MRI and spectroscopy. Magn Reson Med, 1990, 14: 330-346

12 Overgaard K, Meden P. Citicoline--the first effective neuroprotectant to be combined with thrombolysis in acute ischemic stroke? J Neurol Sci, 2006, 247: 119-120

13 Minnerup J, Schabitz W R. Multifunctional actions of approved and candidate stroke drugs. Neurotherapeutics, 2009, 6: 43-52

14 Cho H J, Kim Y J. Efficacy and safety of oral citicoline in acute ischemic stroke: Drug surveillance study in 4191 cases. Methods Find Exp Clin Pharmacol, 2009, 31: 171-176

15 Clark W M, Williams B J, Selzer K A, et al. A randomized efficacy trial of citicoline in patients with acute ischemic stroke. Stroke, 1999, 30: 2592-2597

16 Clark W M, Wechsler L R, Sabounjian L A, et al. A phase III randomized efficacy trial of $2000 \mathrm{mg}$ citicoline in acute ischemic stroke patients. Neurology, 2001, 57: 1595-1602

17 Clark W M. Efficacy of citicoline as an acute stroke treatment. Expert Opin Pharmacother, 2009, 10: 839-846

18 Adibhatla R M, Hatcher J F. Citicoline mechanisms and clinical efficacy in cerebral ischemia. J Neurosci Res, 2002, 70: 133-139

19 Sykova E, Nicholson C. Diffusion in brain extracellular space. Physiol Rev, 2008, 88: 1277-1340

Open Access This article is distributed under the terms of the Creative Commons Attribution License which permits any use, distribution, and reproduction in any medium, provided the original author(s) and source are credited. 